\title{
Improve the Nonparametric Image Segmentation with Narrowband Levelset and Fast Gauss Transformation*
}

\author{
Murong Jiang, Yinghao Zhong, Xin Wang, Xiaotong Huang, Ruilin Guo \\ School of Information Science and Engineering, Yunnan University, Kunming, China \\ Email: jiangmr@ynu.edu.cn
}

Received August 8, 2012; revised September 8, 2012; accepted September 16, 2012

\begin{abstract}
Nonparametric method based on the mutual information is an efficient technique for the image segmentation. In this method, the image is divided into the internal and external labeled regions, and the segmentation problem constrained by the total length of the region boundaries will be changed into the maximization of the mutual information between the region labels and the image pixel intensities. The maximization problem can be solved by deriving the associated gradient flows and the curve evolutions. One of the advantages for this method does not need to choose the segmentation parameter; another is not sensitive to the noise. By employing the narrowband levelset and Fast Gauss Transformation, the computation time is reduced clearly and the algorithm efficiency is greatly improved.
\end{abstract}

Keywords: Nonparametric Image Segmentation; Mutual Information; Narrowband Levelset; Fast Gauss Transformation

\section{Introduction}

In recent years, the image segmentation technology has been made the great progress with many mathematical analytic methods like Partial Differential Equation widely used in it. Among these methods, the non-edge profile model (short as CV model) raised by Chan and Vese [1] is the most famous curve evolution combing with Mumford-Shah function and the levelset method. In addition, Caselles and Catte et al. [2] discussed geometric model based on the geodetic active contours, it can extract the smooth shape target and test a few outlines at the same time. Caselles, Kimmel et al. [3] and N. Paragios, R. Deriche et al. [4] promoted the geodetic active contour model based on the active contours can evolve the outline splitting and merging naturally along with the time changes and detect the internal and external boundaries for many objects according to the image internal geometry measures. These methods are efficiently, but there still exist some difficulties in the computation. The main reason is the parameter selection. For the different style image, choosing the different parameters will obtain the different qualities, and the parameter should be computed for many times in order to get the needed result, and the suitable parameter values usually depend on someone's

${ }^{*}$ This work has been supported by Chinese NSF Grant No. 11161055 and Yunnan NSF Grant No. 2008PY034. experiences. There are some researches using the information theory into the curve evolution. For example, Unal [5] used the information theoretic active polygons to produce the texture segmentation, the carve evolution is based on the image region information with simply statistics such as the mean or the variance. Sun Da et al. [6] estimated the probability density gradient to detect the image edge according to the field edge point's gradient located in the opposite directions. F. Liu [7] improved the traditional segmentation method with combining the largest mutual information theory and the image histogram.

In this paper, we focus on the implementation of the nonparametric method based on the mutual information developed by J. Kim et al. [8]. In this method, the segmentation problem is developed as the maximization of the mutual information between the region label and the image pixel intensities, subject to a constraint on the total length of the region boundaries. During the iterations, the segmentation result is approached by deriving the associated gradient flow and the curve evolution techniques, extract the target without any parameter given first. But estimating the mutual information between the whole region labels and all the image pixel values in each iteration leads a large amount of calculation. Therefore, we use the narrowband levelset to reduce the computation pixels and use the Fast Gauss Transformation to reduce 
the time consumption for estimating the mutual information. Compared with some popular methods include boundary-based, region-based segmentation, this method does not have the parameter selection problem, and it is also not sensitive to the noise in the image. The algorithm efficiency is greatly improved, and it is more suitable to be used in solving the practical problem.

\section{Nonparametric Method Based on the Mutual Information}

\subsection{Mutual Information between the Image Intensity and the Label}

We review the mutual information between the image intensity and the label which provided by J. Kim et al. [8].

Given an image $I(x), \quad x \in \Omega \subset R^{2}, G(x)$ denotes the image intensity at pixel $x, R_{1}$ and $R_{2}$ denote the two regions which are unknown. Assume that the pixel intensities in each region are independent, identically and distributed (i.i.d), the associated densities $p_{1}$ if $x \in R_{1}$ and $p_{2}$ if $x \in R_{2}$ as follows:

$$
\begin{gathered}
\left\{G(x) \mid x \in R_{1}\right\}^{i . i . d} p_{1} \\
\left\{G(x) \mid x \in R_{2}\right\}^{i . i . d} p_{2}
\end{gathered}
$$

where $p_{1}, p_{2}$ are also unknown.

Given an initial evolution curve $\boldsymbol{C}$, then $\boldsymbol{C}$ may divide the image into two regions, $R_{+}$is the region inside the curve and $R_{-}$is the region outside the curve. Suppose that $R_{+}$corresponds to $R_{1}$ (object region) and $R_{-}$corresponds to $R_{2}$ (background region), then the segmentation problem is to move the curve $C$ such that $R_{+}$and $R_{-}$converge to $R_{1}$ and $R_{2}$ respectively (see Figure 1).

Define the label as follows

$$
L_{C}(x)= \begin{cases}L_{+}, & x \in R_{+} \\ L_{-}, & x \in R_{-}\end{cases}
$$

$L_{C}(x)$ is a binary random variable which depends on the curve $C$. According to the probability, we have

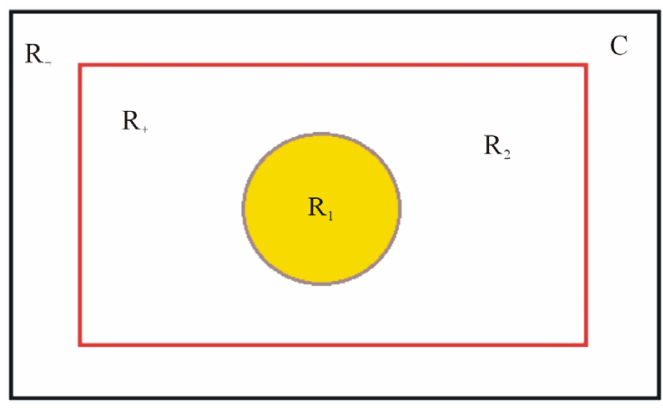

Figure 1. Initial the evolution curve.

$$
\begin{aligned}
& p\left(L_{C}(x)=L_{+}\right)=\frac{\left|R_{+}\right|}{|\Omega|}, \\
& p\left(L_{C}(x)=L_{-}\right)=\frac{\left|R_{-}\right|}{|\Omega|}
\end{aligned}
$$

where $\left|R_{+}\right|$denotes the area of the region $R_{+}$and $\left|R_{-}\right|$ the area of the region $R_{-}$. Therefore, the more accurately the label $L_{C}(x)$ is, the less uncertainty $G(x)$ has. Choose an arbitrary point $X \in \Omega$ and $X$ being in $R_{1}$ or $R_{2}$ is uncertain, then the mutual information $I\left(G(X) ; L_{C}(X)\right)$ is given by

$$
\begin{aligned}
& I\left(G(X) ; L_{C}(X)\right) \\
& =h(G(X))-h\left(G(X) \mid L_{C}(X)\right) \\
& =h(G(X))-p_{\left(G(X) \mid L_{C}(X)=L_{+}\right)} h\left(G(X) \mid L_{C}(X)=L_{+}\right) \\
& \quad-p_{\left(G(X) \mid L_{C}(X)=L_{-}\right)} h\left(G(X) \mid L_{C}(X)=L_{-}\right)
\end{aligned}
$$

where $h(Z)$ is the differential entropy of a continuous random variable $Z$ with support $S$ defined by

$$
h(Z)=-\int_{S} p_{Z}(z) \log p_{Z}(z) \mathrm{d} z
$$

and

$$
\begin{aligned}
& h\left(G(X) \mid L_{C}(X)=L_{+}\right) \\
& =-\frac{1}{\left|R_{+}\right|} \int_{R_{+}} \log \hat{p}_{+}(G(x)) \mathrm{d} x \\
& =-\frac{1}{\left|R_{+}\right|} \int_{R_{+}} \log \left(\frac{1}{\left|R_{+}\right|} \int_{R_{+}} K(G(x)-G(\hat{x})) \mathrm{d} \hat{x}\right) \mathrm{d} x \\
& h\left(G(X) \mid L_{C}(X)=L_{-}\right) \\
& =-\frac{1}{\left|R_{-}\right|} \int_{R_{-}} \log \left(\frac{1}{\left|R_{-}\right|} \int_{R_{-}} K(G(x)-G(\hat{x})) \mathrm{d} \hat{x}\right) \mathrm{d} x
\end{aligned}
$$

where $K(z)=\frac{1}{\sqrt{2 \pi \sigma^{2}}} \mathrm{e}^{-\frac{z^{2}}{2 \sigma^{2}}}$ is the kernel and $\sigma$ is given. The two conditional distributions are given by

$$
\begin{aligned}
& p_{\left(G(X) \mid L_{C}(X)=L_{+}\right)}(z) \\
& =\frac{\left|R_{+} \cap R_{1}\right|}{\left|R_{+}\right|} p_{1}(z)+\frac{\left|R_{+} \cap R_{2}\right|}{\left|R_{+}\right|} p_{2}(z) \\
& p_{\left(G(X) \mid L_{C}(X)=L_{-}\right)}(z) \\
& =\frac{\left|R_{-} \cap R_{1}\right|}{\left|R_{-}\right|} p_{1}(z)+\frac{\left|R_{-} \cap R_{2}\right|}{\left|R_{-}\right|} p_{2}(z)
\end{aligned}
$$

Since the mutual information $I\left(G(X) ; L_{C}(X)\right)$ describes the correlation between $G(X)$ and $L_{C}(X)$, when $R_{+}=R_{1}, R_{-}=R_{2}$, then $C$ is the correct segmentation, and $I\left(G(X) ; L_{C}(X)\right)$ is the maximized. 


\subsection{Energy Functional and Its Computation}

In order to compute the maximum $I\left(G(X) ; L_{C}(X)\right)$, we use the energy functional given as [8]

$$
E(\boldsymbol{C})=-|\Omega| I\left(G(X) ; L_{C}(X)\right)+\alpha \oint_{C} \mathrm{~d} s
$$

where $\oint_{C} \mathrm{~d} s$ is the length of the curve $C, \alpha$ is a scalar parameter. Therefore, the maximization problem of the mutual information $I\left(G(X) ; L_{C}(X)\right)$ subject to a constraint on the total length of the region boundaries $C$ may be changed into the minimization of the energy functional $E(\boldsymbol{C})$.

Let $C(t)$ denotes the curve evolution at time $t$, rewrite the energy functional $E(\boldsymbol{C})$ as

$$
E(\boldsymbol{C}(t))=\int_{R(\boldsymbol{C}(t))} f(x) \mathrm{d} x
$$

where $R(\boldsymbol{C}(t))$ is the region inside the curve $\boldsymbol{C}(t)$. By using the variation principle and employing Equations (3) and (4), we have the gradient flow equation for $E(\boldsymbol{C})$ as [8]

$$
\begin{aligned}
\frac{\partial \boldsymbol{C}}{\partial t}= & {\left[\log \frac{\hat{p}_{+}(G(\boldsymbol{C}))}{\hat{p}_{-}(G(\boldsymbol{C}))}+\frac{1}{\left|R_{+}\right|} \int_{R_{+}} \frac{K(G(x)-G(\boldsymbol{C}))}{\hat{p}_{+}(G(x))} \mathrm{d} x\right.} \\
& \left.-\frac{1}{\left|R_{-}\right|} \int_{R_{-}} \frac{K(G(x)-G(\boldsymbol{C}))}{\hat{p}_{-}(G(x))} \mathrm{d} x\right] \boldsymbol{N}-\alpha \kappa \boldsymbol{N}
\end{aligned}
$$

where $\kappa$ is the curvature of the curve, $N$ is the outward unit normal vector, $-\alpha \kappa N$ is the gradient flow for the curve length penalty, and

$$
\hat{p}_{+}(G(x))=\frac{1}{n} \sum_{i=1}^{n} K\left(G(x)-G\left(x_{i}\right)\right)
$$

is the density estimate at pixel $x$ inside the curve $\boldsymbol{C}$.

We compute the gradient flow Equation (8) with the levelset method. The steps are shown as following:

Step 1. Input an image $I$ and set the initialize curve $C$ according to the image size.

Step 2. Compute the signed distance function $\phi$ as the levelset function with

$$
\begin{cases}\phi(x, y)=d(\boldsymbol{C}, g(x, y)) & \text { if } g(x, y) \text { is inside } \boldsymbol{C} \\ \phi(x, y)=0 & \text { if } g(x, y) \text { is on } \boldsymbol{C} \\ \phi(x, y)=-d(\boldsymbol{C}, g(x, y)) & g(x, y) \text { is outside } \boldsymbol{C}\end{cases}
$$

where $d(\boldsymbol{C}, g(x, y))$ is the Euclidean distance between $\boldsymbol{C}$ and the point $g(x, y)$.

Step 3. Save the points on the curve $C$ into $I_{b}$, the points inside the curve $C$ into $I_{n}$ and outside $C$ into $I_{w}$.

Step 4. Compute the second term $\hat{p}_{+}(G(x))$ noted as $t_{3}$ and the third term $\hat{p}_{-}(G(x))$ noted as $t_{4}$ with

$$
t_{3}(j)=\frac{1}{n_{1}} \sum_{i=1}^{n_{1}} K\left(I_{n}(j)-I_{n}(i)\right)
$$

$$
t_{4}(j)=\frac{1}{n_{2}} \sum_{i=1}^{n_{2}} K\left(I_{w}(j)-I_{w}(i)\right)
$$

where $n_{1}$ is the pixel number of $I_{n}, n_{2}$ is the pixel number of $I_{w}$, and $K(z)=\left(1 / \sqrt{2 \pi \sigma^{2}}\right) \mathrm{e}^{-Z^{2} / 2 \sigma^{2}}$.

Step 5. Compute the first term $\hat{p}_{+}(G(C))$ noted as $S_{1}$, and $\hat{p}_{-}(G(\boldsymbol{C}))$ noted as $S_{2}$ with

$$
\begin{aligned}
& S_{1}(j)=\left(\frac{1}{n_{1}}\right) \sum_{i=1}^{n_{1}} K\left(I_{b}(j)-I_{n}(i)\right) \\
& S_{2}(j)=\left(\frac{1}{n_{2}}\right) \sum_{i=1}^{n_{2}} K\left(I_{b}(j)-I_{w}(i)\right)
\end{aligned}
$$

Step 6. Compute the term $\frac{1}{\left|R_{+}\right|} \int_{R_{+}} \frac{K(G(x)-G(\boldsymbol{C}))}{\hat{p}_{+}(G(x))} \mathrm{d} x$ noted as $L_{2}$ and $\frac{1}{\left|R_{-}\right|} \int_{R_{-}} \frac{K(G(x)-G(C))}{\hat{p}_{-}(G(x))} \mathrm{d} x$ noted as $L_{3}$ with

$$
\begin{aligned}
& L_{2}(j)=\left(\frac{1}{n_{1}}\right) \sum_{i=1}^{n_{1}} K\left(I_{n}(i)-I_{b}(j)\right) / t_{3}(i) \\
& L_{3}(j)=\left(\frac{1}{n_{2}}\right) \sum_{i=1}^{n_{2}} K\left(I_{w}(i)-I_{b}(j)\right) / t_{4}(i)
\end{aligned}
$$

Step 7. Calculate the curvature $\kappa$ and save it in $L_{1}$. Step 8. Renew the levelset function $\phi$ with

$$
\phi^{(i)}=\phi^{(i)}+N\left(\log \frac{S_{1}(i)}{S_{2}(i)}+L_{2}(i)-L_{3}(i)-\alpha^{*} \kappa\right)
$$

If $\left|\phi^{(n)}-\phi^{(n-1)}\right|<\varepsilon$ holds, then stop the computation, else return to Step 3 .

Figure 2 shows the result for the noisy image segmentation implemented on Matlab2009a.

\section{Using the Narrowband Levelset and Fast Gauss Transformation to Improve the Computation Efficiency}

Direct to compute the gradient flow Equation (8) is more expensive. During the iteration, compute $\hat{p}_{+}(G(x))$ and $\hat{p}_{-}(G(x))$ at each pixel $x$ on the curve will take $O\left(n_{1}\right)$ and $O\left(n_{2}\right)$ times, compute the density

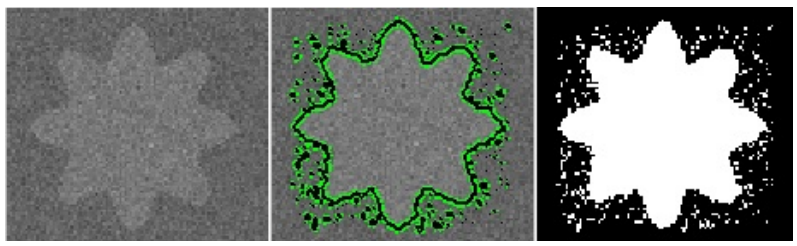

Figure 2. Noisy image segmentation. 
$\hat{p}_{+}(G(\boldsymbol{C}))$ and $\hat{p}_{-}(G(\boldsymbol{C}))$ at all the points on the curve will take $O\left(M\left(\left|R_{+}\right|+\left|R_{-}\right|\right)\right)$time, where $M$ is the number of pixels along the curve. Therefore, compute the gradient flow equation to get the mutual information between the whole region labels and all the image pixel values needs a large amount of calculation. Then, we consider about the narrowband levelset which makes the levelset function update only in the narrowband. Similarly, we use the Fast Gauss Transformation to reduce the time consumption.

\subsection{Narrowband Levelset Method}

Narrowband is a ring surrounding the curve $\boldsymbol{C}$. Narrowband levelset $[9,10]$ is to set the narrowband by choosing a width $\delta$, contracting and expanding the zero levelset curve $\boldsymbol{C}$ with $\delta / 2$ respectively to get the curve $\boldsymbol{C}_{1}$ and $\boldsymbol{C}_{2}$, then the narrowband region is located between the curve $C_{1}$ and $C_{2}$ shown as Figure 3. In each iteration, the computation only be produced on the points in the narrowband, the amount for the renew points is reduced, and the computation quantities is also descend obviously.

Using the narrowband to renew the levelset function is detailed as follows:

1) Initiate the narrowband. Assume the width of the narrowband is $\delta$, then initial the label region, assign a larger negative number to the points inside the curve and a larger positive number to the points outside the curve.

2) Iterate to renew the levelset. After one iteration, set the new function $\phi$ to the curve $C$, check if the curve $\boldsymbol{C}$ is exceed the bounds of the narrowband. If $\boldsymbol{C}$ is cross out of the bound, then reinitialize the narrowband, and compute the levelset function $\phi$ such that the evolution curve $C$ is inside the narrowband. When the computation finished, check whether the terminal condition is satisfied. Once the iteration stops, $\boldsymbol{C}$ is the needed segmentation boundary.

It is easy to see that, different width of the narrowband

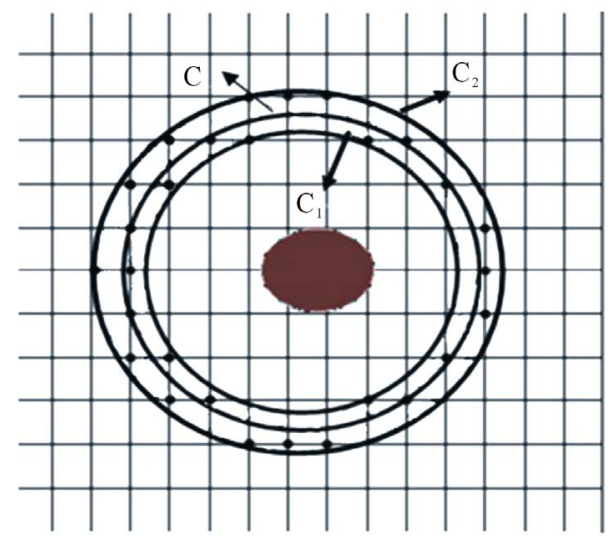

Figure 3. Narrowband. will lead the different efficiency. If the width is too small, the initialization of the narrowband and levelset function need to be replaced many times; and if the width is too large, the number of points will be increased. Both of them will increase the time consumption. Usually, the better width is $7-10$.

\subsection{Fast Gauss Transformation}

Fast Gauss Transformation [11] is using the following weighted Gauss function

$$
G\left(t_{i}\right)=\sum_{j=0}^{N} f_{j} \mathrm{e}^{-\left(t_{i}-s_{j}\right)^{2} / 2 \sigma^{2}}, \quad i=1,2, \cdots, M
$$

where $f_{j}$ is the weights, $s_{j}, j=1,2, \cdots, N$ is the center of the Gauss function, $t_{i}, i=1,2, \cdots, M$ are the goal points. By using the Hermite function: $h_{n}(t)=\mathrm{e}^{-t^{2}} H_{n}(t)$, $t \in R$, where $H_{n}(t)=(-1)^{n} \mathrm{e}^{t^{2}} D^{n} \mathrm{e}^{-t^{2}}, D=\mathrm{d} / \mathrm{d} t$, we have

$$
\begin{aligned}
\mathrm{e}^{-(t-s)^{2} / \sigma} & =\mathrm{e}^{-\left(\left(t-s_{0}\right)-\left(s-s_{0}\right)\right)^{2} / \sigma} \\
& =\sum_{n=0}^{\infty} \frac{1}{n !}\left(\frac{s-s_{0}}{\sqrt{\sigma}}\right)^{n} h_{n}\left(\frac{t-s_{0}}{\sqrt{\sigma}}\right) \\
& =\mathrm{e}^{-\left(t-s_{0}\right)^{2} / \sigma} \sum_{n=0}^{\infty} \frac{1}{n !}\left(\frac{s-s_{0}}{\sqrt{\sigma}}\right)^{n} H_{n}\left(\frac{t-s_{0}}{\sqrt{\sigma}}\right)
\end{aligned}
$$

where $s_{0}$ is the center of the expanding Hermite function. Exchange $s$ and $t$, we also have

$$
\mathrm{e}^{-(t-s)^{2} / \sigma}=\sum_{n=0}^{\infty} \frac{1}{n !} h_{n}\left(\frac{s-t_{0}}{\sqrt{\sigma}}\right)\left(\frac{t-t_{0}}{\sqrt{\sigma}}\right)^{n}
$$

Then the Fast Gauss Transformation is

$$
\begin{gathered}
G\left(t_{i}\right)=\sum_{n=0}^{\infty}\left[\sum_{j=1}^{N} f_{j} h_{n}\left(\frac{s_{j}-t_{0}}{\sqrt{\sigma}}\right)\right] \frac{1}{n !}\left(\frac{t_{i}-t_{0}}{\sqrt{\sigma}}\right)^{n}, \\
i=1,2, \cdots, M
\end{gathered}
$$

Because of the series drop fast and $p \ll \min (m, n)$, the computation amount is also reduced obviously.

Table 1 shows the computation time comparison using four methods for the noisy image (see Figure 4).

From Table 1, we see that the time produced by the levelset is the biggest, and the time for the improved algorithm with the narrowband levelset and Fast Gauss Transformation is the lowest, which means the efficiency of the improved algorithm.

\section{Experimental Results}

\subsection{Compare with Other Segmentation Methods}

Threshold segmentation is a simple but effective solution for most segmentation problems. If the image has some 

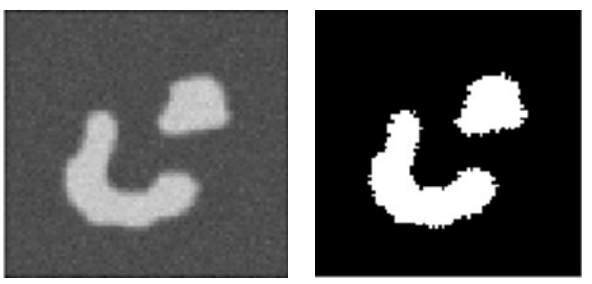

Figure 4. Noisy image segmentation.

Table 1. Computation time using four methods.

\begin{tabular}{cc}
\hline Methods & $\begin{array}{c}\text { Computation Time } \\
\text { (seconds) }\end{array}$ \\
\hline Levelset & 445.3570 \\
Narrowband Levelset & 206.6600 \\
Fast Gauss Transformation & 188.1980 \\
Narrowband and Fast Gauss Transformation & 157.9550 \\
\hline
\end{tabular}

noise, it is difficulty to choose a suitable threshold, one need to produce many experiments to get a better threshold. Region-based segmentation is also sensitive to the noise. Figure 5 shows the results for the noisy image segmentation by using the threshold, region-based and nonparametric methods.

Obviously, for the noisy image, due to the noises located randomly in the whole region, the threshold method can remove some noise but blur the object, and the region-based method can not find the accurately object boundary, but the nonparametric method can get the needed result, although there are some small noisy points in the result image.

\subsection{Several Experiments}

We present some experiment results on the synthetic images.

First, we perform an experiment on an image sized $128 \times 128$ with four synthetic objects. It's difficult for the traditional methods to deal with the noisy image, as they have to filter noisy to get smooth image, which will also filter out some useful information, so the errors are inevitable exist. However, seen from experimental result, nonparametric method can get better segmentation result for noisy image. As shown in Figure 6, it costs 170.601 seconds.

Second, we select another noisy image sized $128 \times$ 128. This image is representative for three reasons: first, the noise is on the object boundary; second, there is a narrow concave part in the middle of the object; third, the image contains three dispersive parts. Traditional methods can do little on the image with such features. However, nonparametric method also can get good segmentation results but the time consumption is higher with 2647.57 seconds shown in Figure 7.

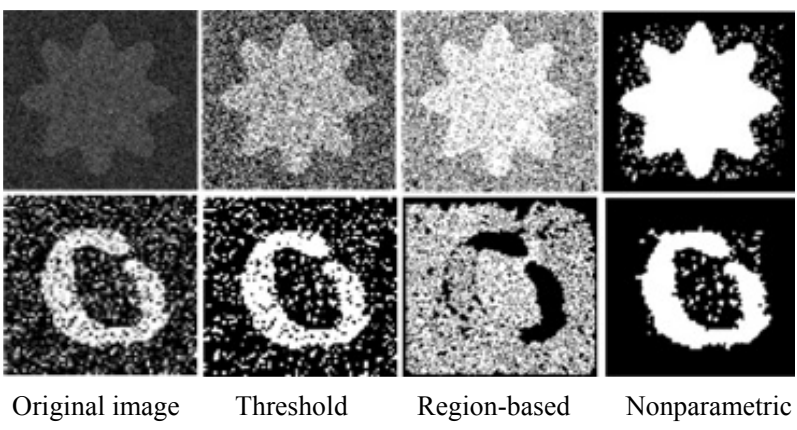

Figure 5. Noisy image segmentation with different methods.

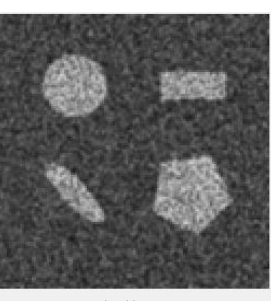

Input image

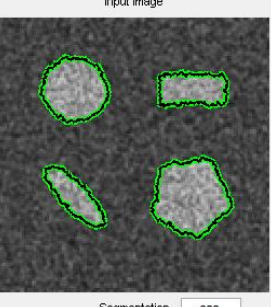

Segmentation 200

Figure 6. Noisy image segmentation.

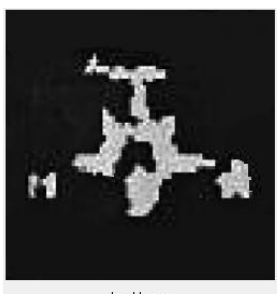

Input limage

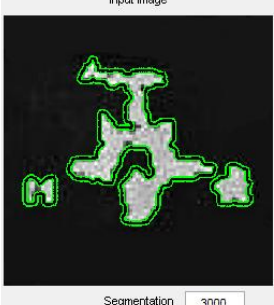

Segmentation 3000

Figure 7. Image with noisy boundary.

Furthermore, we carry out another experiment on a noisy image sized $172 \times 172$ with a narrow gap in the object region. The object likes a character "O" sloped down to the left, and a small break is on the right side. Many traditional methods hardly cross the gap and converge into the inner region. Nonparametric method can separate the object easily, although there are some noisy points left. The segmentation result is better shown in Figure 8. 

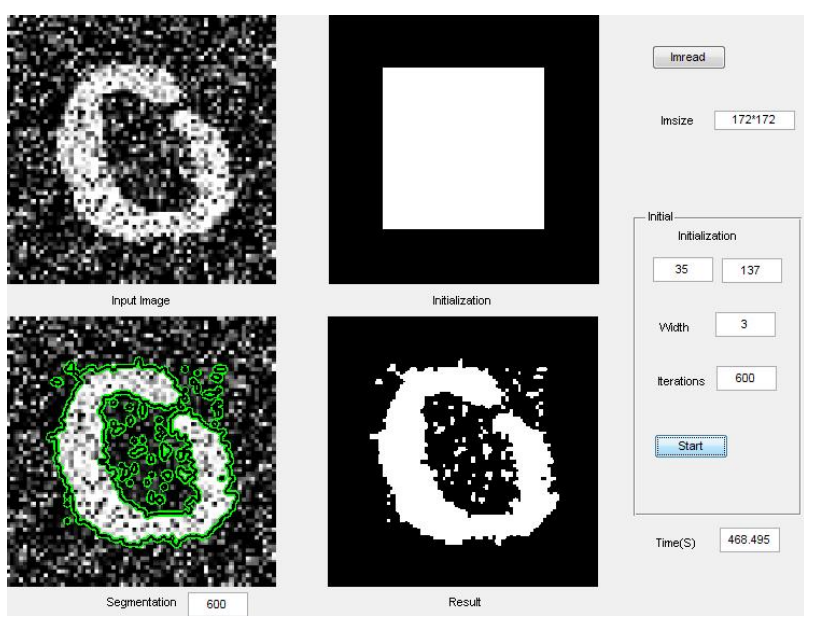

Figure 8. Noisy image segmentation.
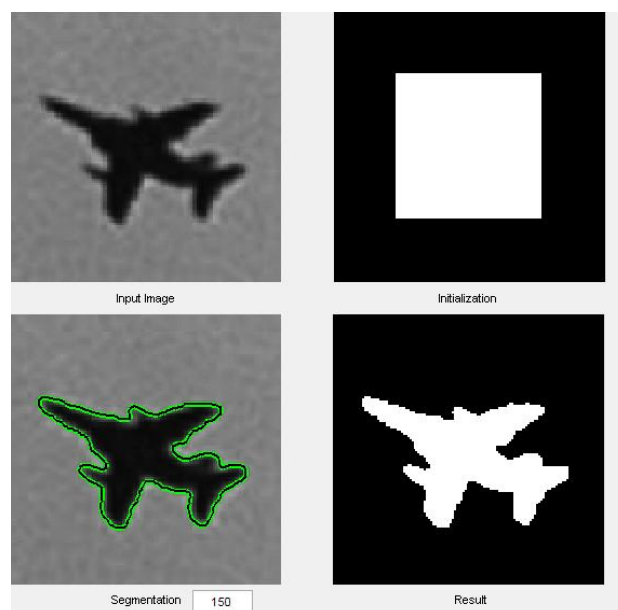

Resut

Figure 9. Image segmentation.

The computation time is 468.495 seconds.

Finally, we select a plane image sized $128 \times 128$, the computation time is 52.6167 seconds (see Figure 9).

From the results shown in Figures 6-9, we see that the improved nonparametric method can process many segmentations with different types, include the larger noisy image, lower contrast gray image, rough boundary image, etc. When the object boundary is smooth, the computing time is shorter; else it will take longer at a reasonable level.

\section{Conclusion}

In this paper, we discuss the nonparametric segmentation method based on the mutual information and its implementation. This method can solve a variety of different types of the image segmentation, and it can get very good results for processing the noise image. However, due to the estimation on the whole image probability density, it makes the calculation increasing greatly. Thus, we discuss an improved method by using the narrowband levelset which only update in the narrow-band, and Fast Gauss Transformation to reduce the iterations. Some experiment show that our improvement can reduce the amount of computation and greatly improve the calculation efficiency. Furthermore, if the implementation can be produced by the parallel computing on the multi-core or cluster computers, the improvement will be upgraded more efficiently.

\section{REFERENCES}

[1] T. Chan and L. Vese, "Active Contours without Edges," IEEE Transactions on Image Processing, Vol. 10, No. 2, 2001, pp. 266-277. doi:10.1109/83.902291

[2] V. Caselles, F. Catte, T. Col and F. Dibos, "A Geometric Model for Active Contours in Image Processing," $\mathrm{Nu}$ merische Mathematik, Vol. 66, 1993, pp. 1-31. doi:10.1007/BF01385685

[3] V. Caselles, R. Kimmel and G. Sapiro, "Geodesic Active Contours," International Journal of Computer Vision, Vol. 22, No. 1, 1997, pp. 61-79. doi:10.1023/A:1007979827043

[4] N. Paragios and R. Deriche, "Geodesic Active Regions and Level Set Methods for Supervised Texture Segmentation," International Journal of Computer Vision, Vol. 46, No. 3, 2002, pp. 223-247. doi:10.1023/A:1014080923068

[5] G. Unal, A. Yezzi Jr. and H. Krim, "Information-Theoretic Active Polygons for Unsupervised Texture Segmentation," International Journal of Computer Vision, Vol. 62, No. 3, 2002, pp. 199-220. doi:10.1007/s11263-005-4880-6

[6] D. Sun, J. Liu and X. Tang, "Edge Detection Based on Density Gradient," Chinese Journal of Computers, Vol. 32, No. 2, 2009, pp. 299-307.

[7] F. Liu, "Medical Image Fine Segmentation Based on the Mutual Information," China Foreign Medical Treatment, No. 2, 2010, pp. 186-187.

[8] J. Kim, J. W. Fisher, A. Yezzi, M. Cetin and A. S. Willsky, "A Nonparametric Statistical Method for Image Segmentation Using Information Theory and Curve Evolution," IEEE Transactions on Image Processing, Vol. 14, No. 10, 2005, pp. 1486-1502. doi:10.1109/TIP.2005.854442

[9] J. A. Sethian, "Level Set Methods: Evolving Interfaces in Geometry, Fluid Mechanics, Computer Vision, and Material Science," Cambridge University Press, Cambridge, 1996.

[10] D. Adalsteinsson and J. A. Sethian, "A Fast Level Set Method for Propagating Interfaces," Journal of Computer Physics, Vol. 118, No. 2, 1995, pp. 269-277. doi:10.1006/jcph.1995.1098

[11] L. Greengard and J. Sethian, "The Fast Gauss Transform," SIAM Journal on Scientific and Statistical Computing, Vol. 12, No. 1, 1991, pp. 79-94. doi:10.1137/0912004 